Proceedings

\title{
Colorimetric Lab-on-a-Tip Based on a Bimetallic Nanoarchitecture ${ }^{\dagger}$
}

\author{
Annalisa Scroccarello *, Flavio Della Pelle and Dario Compagnone \\ Faculty of Bioscience and Technology for Food, Agriculture and Environment, University of Teramo, Via \\ Renato Balzarini 1, 64100 Teramo, Italy; \\ * Correspondence: ascroccarello@unite.it \\ + Presented at the 1st International Electronic Conference on Biosensors, 2-17 November 2020; Available \\ online: https://iecb2020.sciforum.net/.
}

Published: date

\begin{abstract}
Mono- and multi-metal nanoparticles (MNPs), thanks to their unique and tunable features, still fascinate the analytical sciences, from their widespread use in sensing and biosensing as nanoplasmonic tags or catalysts up to MNPs-decorated surfaces. Here, a $\mu$-Tips decorated with plasmonic-active polymeric films embodying gold/silver nanostructures is presented. The proposed lab-on-a-tip device speed-up the 4-nitrophenol conversion in 4-aminophenol, retaining the performances for more than 10 consecutive measures, acting as an enzyme-like catalyst.
\end{abstract}

Keywords: metal nanoparticles; polydopamine film; self-assembled nanoparticles; nanohybrid; nanodecored; catalyst; 4-nitrophenol

\section{Introduction}

Mono- and multi-metal nanoparticles (MNPs) are more and more protagonists of cutting-edge technology, demonstrating unique potentialities in different fields such as medicine, biotechnology, agriculture, etc. [1]. In particular, MNPs, thanks to their unique and tuneable optical features, formally named localized surface plasmon resonance, have inspired the development of a new generation of optical sensing strategies and biosensors. Among these, colorimetric strategies based on the MNPs formation or aggregation have permitted the selective detection of a plethora of analytes (e.g., ascorbic acid, glucose, polyphenols, pesticides, etc.) giving rise to effective assays [1-6]. Furthermore, rationally synthesized MNPs have been also widely employed in bioanalytical applications as nanotags and catalysts [7-10]. MNPs are often defined as 'nanozyme' because able to catalyze chemical and biochemical reactions with notable effectiveness [7-9], allowing both homogeneous and heterogeneous catalysis [7-9].

The dyes regeneration aims to convert a dye from a non-active to an active stare or from a toxic to a non-toxic form [11]. The 4-nitrophenol (4-NP) is a toxic substance potentially dangerous for the environment widely used in pesticides, drugs, and synthetic dyes production [7-9], besides, the 4$\mathrm{NP}$ is also the product of several enzymatic reactions (phosphatase, glycosidase, etc.). Fortunately, the 4-NP can be converted to 4-aminophenol (4-AP) resulting less-toxic, this conversion can be obtained in presence of sodium borohydride, a strong reducing agent, and can be catalyzed throughout different strategies.

Among the innovative non-enzyme catalyzed, chemically or biologically synthesized MNPs, with different metal composition and structure, are widely studied [7-9,11]. The MNPs catalytic efficiency toward 4-NP reduction has been widely demonstrated, Berahim et al. [7] and Wang et al. [9] reported the catalytic performance of bi- (Au@AgNPs) and mono- (AuNPs) metallic 
nanoarchitectures, whereas Kästner et al. [12] discussed the influence of the surfactant around the MNPs in the catalytic performances.

Commonly, nanozyme catalyzer reported in literature are used in solution as nanodispersions, then the related drawbacks of colloidal MNPs as low stability and storability (due to their tendency to aggregate, precipitate, self-oxidize, etc.) need to consider tracing a limit in large-scale application (e.g., wastewater treatment plant). More important, the nature of the nanodispersions makes the reusability of the nano catalyzer not simple, requiring time-spending washing and recovering steps, that can lead to nanodispersions morphology changing with loss of efficiency.

The adhesive nano-films here proposed are composed of AuNPs and AgNPs self-assembled thanks to polydopamine. The supports modified with such film result able to catalyze the 4-NP conversion up to 10 reaction cycles, allowing the 4-NP colorimetric decay monitoring. The proposed adhesive nanocomposite represents a springboard for the MNPs-based catalyst, potentially projectable in large-scale production, also able to give rise to user-friendly devices as the here reported lab-on-a-tips.

\section{Materials and Methods}

\subsection{Reagents, Materials, Samples and Apparatus}

Dopamine hydrochloride $\left(\mathrm{C}_{9} \mathrm{H}_{11} \mathrm{NO}_{2} \cdot \mathrm{HCl}\right.$; DA), hydrogen tetracholoroaurate $\left(\mathrm{HAuCl}_{4} \cdot 3 \mathrm{H}_{2} \mathrm{O}\right.$, 99.9\%; $\mathrm{Au}(\mathrm{III})$ ), silver nitrate $\left(\mathrm{AgNO}_{3}\right)$, sodium hydroxide $(\mathrm{NaOH})$, trizma hydrochloride $\left(\mathrm{C}_{4} \mathrm{H}_{11} \mathrm{NO}_{3} \cdot \mathrm{HCl}\right)$, 4-nitrophenol $\left(\mathrm{C}_{6} \mathrm{H}_{5} \mathrm{NO}_{3}, 4-\mathrm{NP}\right)$; sodium borohydride $\left(\mathrm{NaBH}_{4}\right)$; and $1 \mathrm{~mL}$ polypropylene laboratory-tips were purchased from Sigma-Aldrich (St. Louis, MO, USA).

Polystyrene Cuvette and Gilson pipette were purchased from Sigma-Aldrich (St. Louis, MO, USA). Spectrophotometric measurements were carried out using a JENWAY 6400 Spectrophotometer from Barlworld Scientific (Staffordshire, UK).

\subsection{Metal Nanocomposite Fabrication in Laboratory-Tip}

The metal-based nanocomposite formation was performed in $1 \mathrm{~mL}$ laboratory $\mu$-tips. The labtips decoration was performed according to Scroccarello et al. [13,14], with some modification. A schematization of the whole process is reported in Figure 1.

\subsubsection{PDA Film Self-Assembly}

DA at a concentration of $0.5 \mathrm{~g} \mathrm{~L}^{-1}$ was prepared in Trizma buffer $(10 \mathrm{mM}, \mathrm{pH} 8.5)$ and freshly used to fill lab-tips (800 $\mu \mathrm{L}$ per tip). DA polymerization was carried out in static condition, in a white incubation room, at $25 \pm 2{ }^{\circ} \mathrm{C}$, under a light source ( $20 \mathrm{~W}$, warm light bulb) placed at $50 \mathrm{~cm}$ of distance for a total polymerization time of $15 \mathrm{~h}$. Finally, the polydopamine (PDA) formation was blocked emptying the tips subsequently washed and rinsed with abundant Milli-Q water. The PDA modified laboratory $\mu$-tips ( $\mu$-T@PDA) were let dry at room temperature.

\subsubsection{Gold and Silver Nanodecoration}

- $\quad$ AuNPs decoration: the PDA modified $\mu$-tips were filled with $800 \mu \mathrm{L}$ of $100 \mu \mathrm{M} \mathrm{Au}(\mathrm{III})$ aqueous solution and incubated in the dark at room temperature for $8 \mathrm{~h}$. The reaction was blocked emptying the tips subsequently washed and rinsed with abundant Milli-Q water. The AuNPs decorated $\mu$-T@PDA were let dry at room temperature.

- AgNPs decoration: the AuNPs decorated $\mu$-T@PDA ( $\mu$-T@Au) were filled with $740 \mu \mathrm{L}$ of Milli$\mathrm{Q}$ water and $40 \mu \mathrm{L}$ of $20 \mathrm{mM} \mathrm{AgNO}$, afterward, $20 \mu \mathrm{L}$ of $4 \mathrm{M} \mathrm{NaOH}$ was added to trig the reaction (final volume $800 \mu \mathrm{L}$ ). The reaction mix was orbitally shaken (SSL1, Stuart equipment, Belfast, UK150) at $150 \mathrm{rpm}$ at room temperature, in the dark, for $4 \mathrm{~h}$. The reaction was blocked emptying the tips subsequently washed and rinsed with abundant Milli-Q water. The AgNPs decorated $\mu-\mathrm{T} @ \mathrm{Au}(\mu-\mathrm{T} @ \mathrm{Au} @ \mathrm{Ag})$ were let dry at room temperature. 
- $\quad A u @ A g N P s$ decoration: for the bimetallic nanocomposite formation, the previous two steps were performed consecutively.

\subsection{4-Nitrophenol Reduction}

The 4-nitrophenol (4-NP) reduction was performed directly in the modified $\mu$-Tips $(\mu$-T@Au, $\mu$ $\mathrm{T} @ \mathrm{Ag}$, and $\mu-\mathrm{T} @ \mathrm{Au} @ \mathrm{Ag}$ ) in presence of $\mathrm{NaBH}_{4}$. The reaction mix was directly prepared in cuvette with $980 \mu \mathrm{L}$ of $0.01 \mathrm{M} \mathrm{NaBH}_{4}$ and $10 \mu \mathrm{L}$ of $4-\mathrm{NP}$ at different concentrations (final volume $1 \mathrm{~mL}$ ). Then, the mix of reaction was sucked in the $\mu$-T through Gilson pipette and incubated in dark condition. The 4-NP color decay evolution kinetics (conversion at 4-AP, colorless) was monitored in cuvette through absorbance recording every $5 \mathrm{~min}$ at $400 \mathrm{~nm}\left(t_{n}\right)$ against the absorbance at time $0\left(t_{0}\right)$, according to the following equation:

$$
A b s_{t_{n} / t_{0}}=A b s_{t_{n}} / A b s_{t_{0}}
$$

Finally, for each tip, the dose-response curve was built up measuring the 4-NP decoloration (D\%, see equation below), after 15 min of incubation, against increasing amounts of 4-NP.

$$
D(\%)=\left(\left(A b s_{t_{0}}-A b s_{t_{15}}\right) / A b s_{t_{0}}\right) \times 100
$$

\section{Results and Discussion}

\section{1. $\mu$-Tip-Surfaces Nanodecoration}

The aim of this work was the development of a reusable and smart nano-catalyst system for the monitoring and degradation of 4-NP. $1 \mathrm{~mL}$ laboratory $\mu$-tips $(\mu$-T) were modified with a PDA film and subsequently decorated with AuNPs, AgNPs, and a bimetallic Au@AgNPs nanocomposite, respectively, and the tips catalytic performance were evaluated against 4-NP reduction (Figure 1). Dopamine (DA) and metal-precursors concentrations, $\mathrm{pH}$, growth time of both the polymer and the nanoparticles (see Section 2.2) were selected according to our group's previous works [13,14]. In brief, the formation of the PDA thin-film onto the $\mu$-T surface was achieved through a DA polymerization in mild alkaline condition, the resulting polymer, thanks to its redox-active chemistry, allowed the MNPs (AuNPs and AgNPs) growing and anchoration. The PDA chemistry allows a strong adhesivity permitting an in-situ nanocomposite formation onto different surfaces. Then, exploiting the PDA features reproducible nanometallic-based films were obtained onto several substrates (Pasteur pipette, Eppendorf, ELISA plate, cuvette, tips, etc.) composed of different materials (glass, polyethylene terephthalate, etc.) (Figure 1).

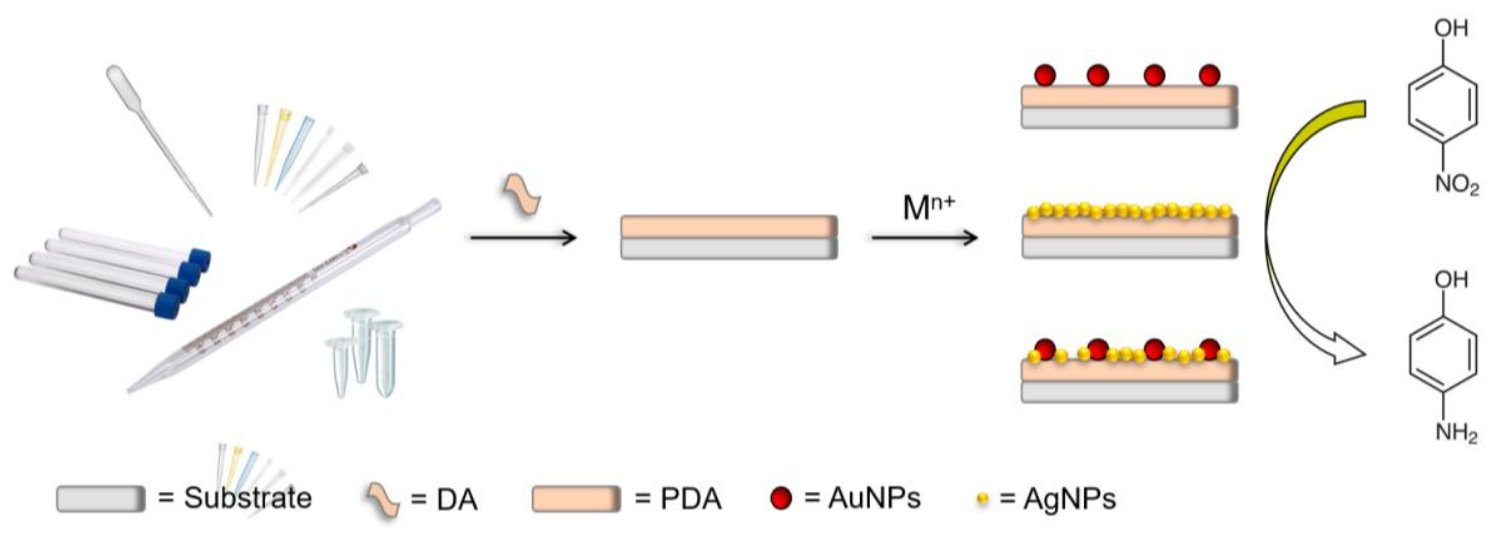

Figure 1. Schematization of lab-devices nanodecoration. 


\subsection{Atalytic Reduction of 4-Nitrophenol by Using Nanodecorated $\mu$-Tips}

As illustrated in Figure 2, the 4-NP reduction by the nanodecorated $\mu$-Tips occurs in three main steps: (i) reaction mix absorbance reading at time zero $(\lambda=400 \mathrm{~nm}$ ); (ii) reaction mix suctions in the $\mu$-Tips and incubation (15 min), (iii) reacted mix color decay reading $(\lambda=400 \mathrm{~nm}$ ).

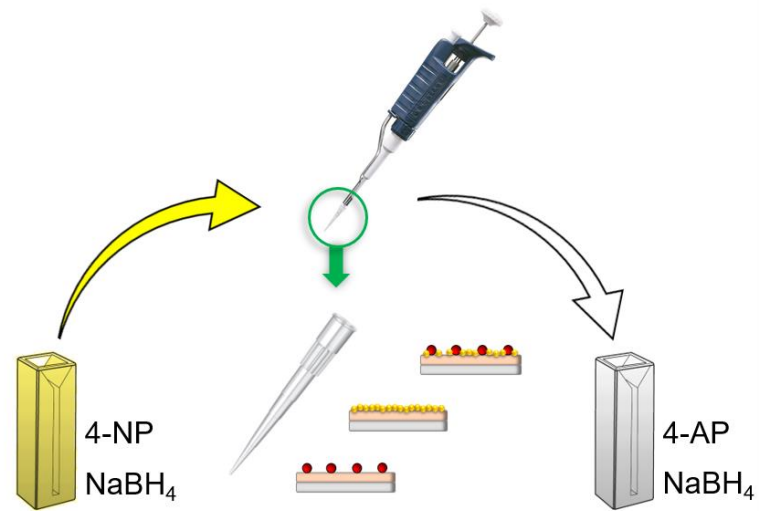

Figure 2. Schematization of the 4-NP reduction at 4-AP, associated with a colorimetric decay, driven by the catalytic action of the nanodecorated $\mu$-Tips.

As reported in Figure 3a, it is possible to notice how at the same reaction conditions, the reduction rate of the three different nanodecorated $\mu$-Tips results significantly different. Noteworthy by using the undecorated polypropylene and PDA decorated $\mu$-Tips no decoloration occurs, confirming the catalytic role of the nanodecorated surfaces towards the 4-NP reduction (Figure 3a). Among the different decorating $\mu$-Tips the $\mu$-T@Au@Ag result the most catalytic, followed by the $\mu$ T@Ag and the $\mu-\mathrm{T} @ \mathrm{Au}$. The $\mu-\mathrm{T} @ \mathrm{Au} @ \mathrm{Ag}$ allows the 4-NP complete reduction (decoloration) in 25 $\min$.

The obtained data results totally in accordance with literature, where was demonstrated how multi-metallic nanodispersions improve reaction rates compared to single metals. Besides, it is well recognized how the catalytic activity towards the 4-NPs reduction is strictly related to the size, shape and electron density of the employed nanomaterials [7]. In the case of the $\mu-\mathrm{T} @ \mathrm{Au} @ \mathrm{Ag}$ is noticeable that the catalytic performance of the $\mu-\mathrm{T} @ \mathrm{Au} @ \mathrm{Ag}$ is ascribable to the catalyst behavior of the Au and AgNPs (Figure 3a). In our case, for the $\mu-\mathrm{T} @ \mathrm{Au} @ \mathrm{Ag}$ the superior catalytic performance can be ascribed to the nano $\mathrm{Au}$ and $\mathrm{Ag}$ catalytic synergistic action, nevertheless, in order to better understand the $\mathrm{Au}$ and $\mathrm{Ag}$ role an accurate modeling and parameterization of the kinetic curves is required.

(a)

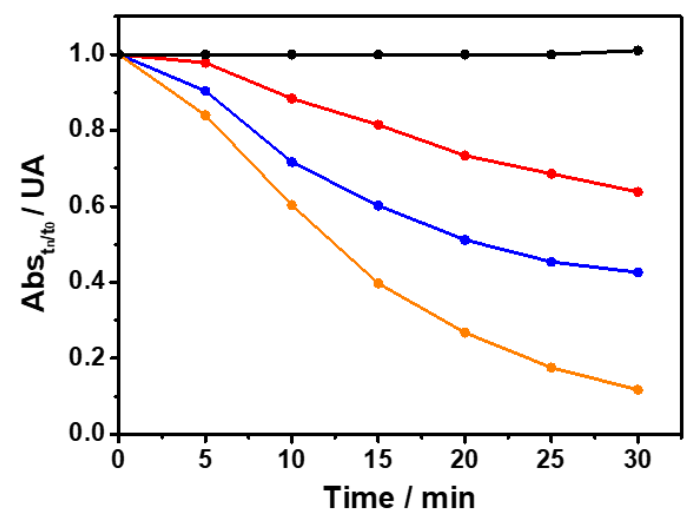

(b)

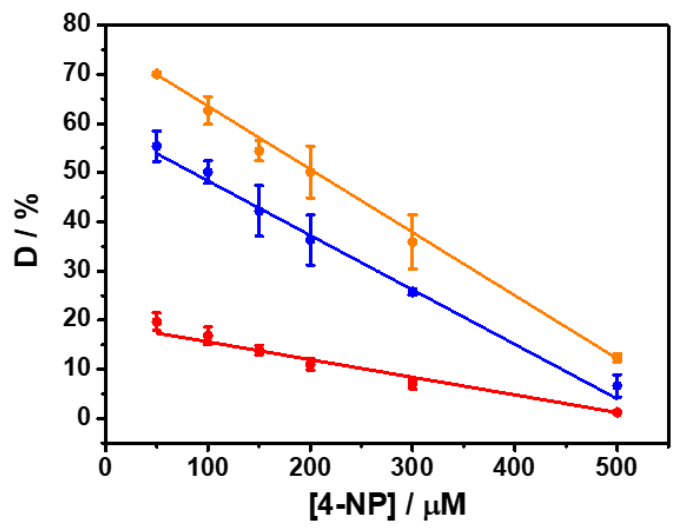


Figure 3. Kinetic curves of 4-NP $(100 \mu \mathrm{M})$ reduction at 4-AP in the presence of $0.01 \mathrm{M} \mathrm{NaBH}_{4}$ by using the $\mu$-T@PDA (black curve), $\mu-\mathrm{T} @ \mathrm{Au}$ (red curve), $\mu$-T@Ag (blue curve), and $\mu$-T@Au@Ag (orange curve). Dose-response curves of 4-NP carried out in presence of $0.01 \mathrm{M} \mathrm{NaBH}_{4}$ by using the $\mu$-T@Au (red curve), $\mu-\mathrm{T} @ \mathrm{Ag}$ (blue curve), and $\mu-\mathrm{T} @ \mathrm{Au} @ \mathrm{Ag}$.

Finally, to study the 4-NP concetration effect on the catalytic reaction, and to obtain quantitative monitoring of the 4-NP decay, dose-response curves in the range of 50-500 $\mu \mathrm{M}$ were built-up with the nanodecorated $\mu$-Tips (Figure $3 b$ ). As expected, the $\mu$-Tips previously observed catalytic behaviors results resumed in the dose response-curves slopes (see Table 1), where the slope obtained with the $\mu-\mathrm{T} @ \mathrm{Au} @ \mathrm{Ag}$ result the highest, followed by the $\mu-\mathrm{T} @ \mathrm{Ag}$ and the $\mu-\mathrm{T} @ \mathrm{Au}$. As expected,no dose-response curve was obtained with the PDA-modified $\mu$-Tip. The proposed devices result usable up to 10 consecutive 'measures' ensuring the same catalytic activity (RSD $\leq 5 \%$ ), demonstrating a significant advantage compared to MNPs-based catalysis in colloidal solutions. The devices are storable at room temperature for one month without significant loss in the catalyticity.

Table 1. Dose-response curve parameter obtained with the $\mu-T @ A u, \mu-T @ A g$, and $\mu-T @ A u @ A g$.

\begin{tabular}{ccccc}
\hline$\mu$-Tip & $\begin{array}{c}\text { Concentration Range } \\
(\mu \mathbf{M})\end{array}$ & Linear Equation & Slope & R $^{2}$ \\
\hline$\mu-\mathrm{T} @ \mathrm{Au} @ \mathrm{Ag}$ & $50-500$ & $\mathrm{y}=-0.13(0.00) \mathrm{x}+76.40(0.30)^{1}$ & -0.13 & 0.999 \\
$\mu-\mathrm{T} @ \mathrm{Ag}$ & $50-500$ & $\mathrm{y}=-0.11(0.01) \mathrm{x}+59.47(1.62)^{1}$ & -0.11 & 0.998 \\
$\mu-\mathrm{T} @ \mathrm{Au}$ & $50-500$ & $\mathrm{y}=-0.04(0.00) \mathrm{x}+19.18(0.95)^{1}$ & -0.04 & 0.985 \\
\hline
\end{tabular}

${ }^{1}$ All the dose response curve was performed in triplicates $(n=3)$.

\section{Conclusions}

Here, a nanodecoration strategy to modify laboratory $\mu$-tips has been proposed for the first time. The proposed procedure allows decorating $\mu$-tips surfaces with AuNPs, AgNPs and their nanocomposites. The superior catalytic activity of the bimetallic films (t@PDA@Au@Ag) for the 4-NP reduction was demonstrated. The here proposed lab-on-a-tip catalyzer result reusable (up to 10 times) elegantly overcoming the colloidal MNPs limitations, also ensuring a 4-NP quantitatively monitoring. The here proposed nanodecorated-films results versatile and potentially usable onto different supports allowing tailor-nano decorations, proposing itself as new and useful analytical tools potentially suitable for catalysts and biosensors developments.

\section{References}

1. Della Pelle, F.; Compagnone, D. Nanomaterial-Based Sensing and Biosensing of Phenolic Compounds and Related Antioxidant Capacity in Food. Sensors 2018, 18, 462, doi:10.3390/s18020462.

2. Della Pelle, F.; Scroccarello, A.; Sergi, M.; Mascini, M.; Del Carlo, M.; Compagnone, D. Simple and rapid silver nanoparticles based antioxidant capacity assays : Reactivity study for phenolic compounds. Food Chem. 2018, 256, 342-349, doi:10.1016/j.foodchem.2018.02.141.

3. Della Pelle, F.; Vilela, D.; Gonzàlez, M.C.; Lo Sterzo, C.; Compagnone, D.; Del Carlo, M.; Escarpa, A. Antioxidant capacity index based on gold nanoparticles formation. Application to extra virgin olive oil samples. Food Chem. 2015, 178, 70-75, doi:10.1016/j.foodchem.2015.01.045.

4. Della Pelle, F.; Sergi, M.; Del Carlo, M.; Compagnone, D.; Escarpa, A. Gold Nanoparticles-based ExtractionFree Colorimetric Assay in Organic Media: An Optical Index for Determination of Total Polyphenols in Fat-Rich Samples. Anal. Chem. 2015, 87, 6905-6911, doi:10.1021/acs.analchem.5b01489.

5. Della Pelle, F.; Scroccarello, A.; Scarano, S.; Compagnone, D. Silver nanoparticles-based plasmonic assay for the determination of sugar content in food matrices. Anal. Chim. Acta 2019, 1051, 129-137, doi:10.1016/j.aca.2018.11.015.

6. Yu, L.; Li, N. Noble metal nanoparticles-based colorimetric biosensor for visual quantification: A mini review. Chemosensors 2019, 7, doi:10.3390/chemosensors7040053.

7. Berahim, N.; Basirun, W.; Leo, B.; Johan, M. Synthesis of Bimetallic Gold-Silver (Au-Ag) Nanoparticles for the Catalytic Reduction of 4-Nitrophenol to 4-Aminophenol. Catalysts 2018, 8, 412, doi:10.3390/catal8100412. 
8. Choi, Y.; Choi, M.J.; Cha, S.H.; Kim, Y.S.; Cho, S.; Park, Y. Catechin-capped gold nanoparticles: Green synthesis, characterization, and catalytic activity toward 4-nitrophenol reduction. Nanoscale Res. Lett. 2014, 9, 1-8, doi:10.1186/1556-276X-9-103.

9. Wang, J.G.; Hua, X.; Li, M.; Long, Y.T. Mussel-inspired polydopamine functionalized plasmonic nanocomposites for single-particle catalysis. ACS Appl. Mater. Interfaces 2017, 9, 3016-3023, doi:10.1021/acsami.6b14689.

10. Kumar, S.; Sharma, R.K. Work Function Based Catalytic Activity of Metallic Nanoparticles for Dye Degradation. Catal. Lett. 2019, doi:10.1007/s10562-019-02744-4.

11. Baruah, D.; Goswami, M.; Yadav, R.N.S.; Yadav, A.; Das, A.M. Biogenic synthesis of gold nanoparticles and their application in photocatalytic degradation of toxic dyes. J. Photochem. Photobiol. B Biol. 2018, 186, 51-58, doi:10.1016/j.jphotobiol.2018.07.002.

12. Kästner, C.; Thünemann, A.F. Catalytic Reduction of 4-Nitrophenol Using Silver Nanoparticles with Adjustable Activity. Langmuir 2016, 32, 7383-7391, doi:10.1021/acs.langmuir.6b01477.

13. Scroccarello, A.; Della Pelle, F.; Ferraro, G.; Fratini, E.; Tempera, F.; Dainese, E.; Compagnone, D. Plasmonic active film integrating gold/silver nanostructures for $\mathrm{H} 2 \mathrm{O} 2$ readout. Talanta 2021, 222, 121682, doi:10.1016/j.talanta.2020.121682.

14. Scroccarello, A.; Della Pelle, F.; Fratini, E.; Ferraro, G.; Scarano, S.; Palladino, P.; Compagnone, D. Colorimetric determination of polyphenols via gold nanoseeds decorated polydopamine film. Microchim. Acta 2020, 187, 1-10, doi:10.1007/s00604-020-04228-4.

Publisher's Note: MDPI stays neutral with regard to jurisdictional claims in published maps and institutional affiliations.

(C) 2020 by the authors. Submitted for possible open access publication under the terms and conditions of the Creative Commons Attribution (CC BY) license (http://creativecommons.org/licenses/by/4.0/). 